\title{
Refluxo gastroesofágico participando da cascata cognitiva do pânico
}

\author{
Gastroesophageal reflux participating on panic cognitive cascade
}

Kalil Duailibi', Rafael Brandes Lourenço², Elisa Poleza Mello², Marcelo Gontijo Magalhães², Geraldo Teles Machado Netto², Mariana Campoy Noé Diaz², Thaísa Saddi Tannous²

\section{RESUMO}

O transtorno do pânico (TP) é um transtorno ansioso não-fóbico que acomete de 1,5\% a 4\% da população mundial. É caracterizado por ataques imotivados de mal-estar psíquico e sintomas somáticos, além de ansiedade antecipatória à crise, com prejuízo funcional ao indivíduo. O objetivo deste relato de caso é descrever a associação entre transtorno do pânico e doença do refluxo gastroesofágico (DRGE). MCL, 25 anos, apresentava crises de pânico frequentes, pouco responsivas ao tratamento durante 6 meses, mesmo com readequação da farmacoterapia. Iniciou-se investigação, sendo fechado o diagnóstico de DRGE, cujo tratamento culminou em remissão das crises de pânico. A dor torácica aguda da DRGE era interpretada como ameaça proximal, ocasionando dúvidas sobre passar mal e hiperventilação, servindo como gatilho da cascata cognitiva do pânico, no mesencéfalo dorsal. A inflamação da mucosa esofágica funciona como ameaça distal, estimulando a amígdala e causando ansiedade antecipatória, mantendo a elevação dos hormônios de estresse. Segundo o modelo de Deakin-Graeff, embora a 5-HT iniba o ataque de pânico e facilite a ansiedade antecipatória, no TP esta última é estimulada por meio do núcleo dorsal da rafe. Portanto, casos que incluem a associação TP e DRGE devem ser mais bem examinados, para que haja diagnóstico e tratamento adequados.

\section{ABSTRACT}

Panic disorder (PD) is a non phobic anxiety disorder that affects 1,5 to 4\% worldwide. It is characterized by unmotivated acute attacks, with mental and somatic symptoms, and by an anxiety which precedes the crises, resulting in functional disturbance. The objective of this case study is to describe the association between PD and gastroesophageal reflux (GR). MCL, 25 years, presented with frequent panic crises, with low response to the therapy for 6 months, even after modification of medication doses. Investigation was carried out and diagnoses defined as GR. The treatment resulted with elimination of the panic crises. The acute thoracic pain of the GR is interpreted as a near threat, causing uncertainty regarding an unwell feeling with hyperventilation, acting as a trigger of the panic cognitive cascade, in the dorsal mesencephalon. The esophagic mucosa inflammation is interpreted as a distal threat, stimulating the amygdale and generating antici-

Faculdade de Medicina da Universidade de Santo Amaro (Unisa).

1 Núcleo de Saúde Mental da Faculdade de Medicina da Universidade de Santo Amaro (Unisa), Psiquiatria da Faculdade de Medicina da Unisa. 


\section{Keywords}

Panic disorder, panic

gastroesophageal reflux. pated anxiety, increasing stress hormones. According to Deakin-Graeff model, even though 5HT inhibits the panic attack and stimulates the anticipated anxiety, the panic disorder stimulates the latter through dorsal raphe nucleus. In conclusion, the association between PD and GR should be better identified in order that the correct diagnosis and adequate treatment is achieved.
O transtorno do pânico (TP) é um transtorno mental cuja prevalência varia entre $1,5 \%$ e $4 \%$ da população mundial, sendo mais frequente em mulheres. Tem início, normalmente, entre os 15 e os 19 anos de idade, acometendo, principalmente, a faixa dos 25 anos'.

É classificado pelo DSM-IV-TR como transtorno de ansiedade paroxístico e não-fóbico, que se caracteriza por sensação de mal-estar físico e psíquico agudo, acompanhado por, pelo menos, quatro sintomas somáticos ou cognitivos, como dor torácica, palpitações, tremores, dispnéia, sudorese, sensação de asfixia, tremores, parestesias, desrealização e despersonalização, medo de enlouquecer ou morrer. Para confirmar o diagnóstico, ao menos um dos episódios deve ocasionar ao paciente, por um mês, preocupações acerca do ataque e da possibilidade de novos ataques, ou mudanças de comportamento a eles relacionadas ${ }^{2}$.

O TP é altamente responsivo ao tratamento, possuindo bom prognóstico. O tratamento recomendado, a longo prazo, inclui o uso de antidepressivos e psicoterapia breve, e a curto prazo, o uso de benzodiazepínicos ${ }^{3}$.

Acredita-se que o pânico corresponda à vigorosa reação de fuga evocada por perigo muito próximo, como predador que se aproxima ou por dor intensa aguda ${ }^{4}$. Estes agentes teriam papel de gatilho, iniciando as crises $^{5}$.

No TP, também existe ansiedade antecipatória à crise, que pode ser interpretada como resposta à ameaça distante, estando relacionada com seu prognóstico e sua evolução6.

A doença do refluxo gastroesofágico (DRGE) atinge cerca de $12 \%$ da população adulta brasileira. O DRGE é causado fisiologicamente pelo relaxamento do esfíncter esofágico inferior, por ocasião da distensão do fundo gástrico durante a alimentação. Torna-se patológico quando há mudança na frequência, no conteúdo ou na quantidade do refluxo. Nesses casos, o paciente pode apresentar sintomas típicos, como pirose e regurgitação ácida, ou atípicos, como rouquidão ou dor torácica, porquanto cerca de $40 \%$ dos pacientes com DRGE apresentam, em alguma fase da vida, sintomas da doença?.

Foi realizado levantamento bibliográfico nas bases MedLine, PubMed e Lilacs, utilizando-se os descritores em inglês panic disorder e gastroesophageal reflux, em associação, sem nenhum resultado de retorno às pesquisas.

O objetivo deste relato de caso é descrever a associação entre DRGE e TP.

\section{CASO CLÍNICO}

MCL, 25 anos, masculino, estudante, procedente de São Paulo, chegou ao Ambulatório da Psiquiatria com a queixa de "não conseguir ter uma vida normal" (sic). Apresentava crises imotivadas acompanhadas de dor torácica, dispnéia, sudorese, palpitações, sensação de asfixia e medo de morte, com duração de 30 a 40 minutos, por cerca de 1 mês.

Foram prescritos cloridrato de paroxetina $20 \mathrm{mg} / \mathrm{dia}$ e clonazepan $2 \mathrm{mg} / \mathrm{dia}$. Nos 70 dias seguintes permaneceu estável. A dose de paroxetina foi aumentada para $40 \mathrm{mg} /$ dia e iniciou-se psicoterapia breve. Nos 2 meses subsequentes, o quadro manteve-se inalterado. Entre diversas crises referidas, o paciente aludiu especificamente a uma, ligada a episódio pós-prandial, ao deitar, no período noturno.

A investigação dos sintomas de doença do refluxo foi positiva, com presença de outros episódios de pirose e regurgitação ácida, em geral sem ligação com as crises, mas eventualmente associadas. Foi solicitada endoscopia digestiva alta (EDA). A EDA denotou hiperemia de mucosa, e microscopicamente, infiltrado infamatório, apresentando em laudo final esofagite de refluxo não-erosiva. Foi realizado tratamento padrão com inibidor de bomba de prótons. As demais medicações foram mantidas e houve redução considerável do número de crises nos meses subsequentes.

Após 3 meses, o paciente não apresentou nenhuma crise. Realizou-se nova EDA, sem alterações. Atualmente se encontra estável, sem sintomas de refluxo, apenas em uso de $20 \mathrm{mg}$ de paroxetina.

Embora não se tenha comprovado a melhora por meio da aplicação de entrevista clínica estruturada para o DSMIV $(\mathrm{SCID})^{8}$, constatou-se evolução clínica favorável, visto que ao final do tratamento o paciente não mais satisfazia os critérios diagnósticos do DSM-IV para TP.

\section{DISCUSSÃO}

O paciente $M C L$ apresentava crises de ansiedade frequentes, pouco responsivas à terapia preconizada, durante 6 meses, mesmo com elevação das doses dos medicamentos. Uma vez diagnosticada e tratada a DRGE, a evolução do paciente quanto ao quadro de pânico foi notável. Para esclarecer o envolvimento do refluxo, neste caso, deve-se 
abordar o funcionamento cognitivo da cascata do pânico e suas bases neurobiológicas.

É descrita como cascata cognitiva do pânico a sobreposição de reações a estímulo desencadeante, levando a sintomas somáticos cíclicos. Este estímulo inicial desencadeia hiperventilação, por temor em passar mal. O paciente percebe a hiperventilação como sintoma inicial da crise e inicia sentimentos de luta ou fuga. A partir daí, sintomas somáticos incidem, com repetição da cascata e potencialização dos sintomas ${ }^{9}$.

No caso descrito, o DRGE do paciente ocasionava dor torácica, servindo como estímulo desencadeante (gatilho) da cascata do pânico. Não há como saber se a dor torácica sentida pelo paciente em todas as crises se devia a este sintoma atípico da DRGE ou ao próprio quadro de pânico.

Quanto ao caráter neurobiológico do TP, é sugerido pela espontaneidade das crises, pelo estereótipo de suas manifestações clínicas nas diferentes culturas ${ }^{10}$ e por sua influência genética"1. Por meio da reprodução de sintomas de ataques de pânico, de modo específico em laboratório, chegou-se à série de modelos comportamentais e neuroquímicos, seletivos ou não.

Os modelos seletivos são aqueles que iniciam a crise de pânico, como o modelo de sufocação, em que lactato de sódio e $\mathrm{CO}_{2,}$ induzem resposta por alcalose metabólica ${ }^{5}$. Os modelos não seletivos são mais específicos da ansiedade antecipatória. São eles: noraderenérgico (atividade noradrenérgica aumentada), GABAérgico (agonistas inversos aos benzodiazepínicos se ligariam a estes receptores) e serotonérgico ${ }^{12}$. Somente os modelos não-seletivos ativam o eixo hipotálamo-hipófise-adrenal (HHA). Portanto, o ataque de pânico pouco ativaria o eixo HHA, embora haja acentuada ativação simpática ${ }^{10}$.

Quanto ao modelo serotonérgico, desconfia-se que seja o mais decisivo para o TP, em virtude da boa resposta do tratamento com antidepressivos ${ }^{13}$. As evidências indicam duplo papel da serotonina (5-HT) no TP, segundo o modelo de Deakin-Graeff.

Os ataques de pânico refletiriam reações de defesa induzidas por ameaças proximais e teriam relação com o mesencéfalo dorsal10. Por outro lado, a ansiedade antecipatória teria como base ameaças distais, e seria integrada à amígdala. Segundo esta proposta, a 5-HT facilita a ansiedade, mas inibe o pânico. A integração entre os dois sistemas ocorre no núcleo dorsal da rafe, que no TP ativa a amígdala, levando à ansiedade antecipatória4

No caso do pânico induzido pelo DRGE, o refluxo teria tanto papel na ansiedade antecipatória, auxiliando na manutenção das crises a longo prazo e mantendo elevação dos hormônios de estresse, quanto no ataque de pânico, em que a dor torácica aguda seria interpretada como perigo iminente, estimulando o mesencéfalo dorsal.

Não se sabe exatamente se a melhora seria obtida sem a resolução da DRGE, porém, o fato é que o tratamento do quadro de refluxo contribuiu para a cura do paciente. Esta conduta é semelhante a do uso dos benzodiazepínicos: a eliminação da ansiedade antecipatória e o impedimento da precipitação de novas crises de pânico ${ }^{14}$. Deve-se ressaltar que, embora este tratamento tenha grande importância, a melhor abordagem do TP continua sendo a estabilização serotonérgica, alcançada com o uso de antidepressivos ${ }^{3}$.

\section{CONCLUSÃO}

Casos que incluem a associação entre DRGE e TP devem ser mais bem examinados, para que haja diagnóstico correto e tratamento adequado.

\section{REFERÊNCIAS}

1. Kaplan HI, Sadock BJ. Transtorno do pânico e agorafobia. In: Kaplan HI, Sadock BJ. Compêndio de psiquiatria. Porto Alegre: Artes Médicas; 1997. p. 418-25.

2. American Psychiatric Association. Diagnostic and Statistical Manual of Mental Disorders (DSM-IV) - text revision. 4. ed. Washington, DC: APA; 2002.

3. Savóia MG. Tratamento combinado do transtorno de pânico: farmacológico e psicoterápico/Combined pharmacological and psycotherapic treatment in panic disorder. Rev Psiquia$\operatorname{tr} C \operatorname{lin} .2001 ; 28(1): 35-8$.

4. Graeff FG. Serotonina, matéria cinzenta periaquedutal e transtorno do pânico/Serotonin, periaqueductal gray matter and panic disorder. Rev Bras Psiquiatr. 2003; 25 Suppl 2:42-45.

5. Valença AM, Nardi AE, Nascimento I, Mezassalma MA, Lopes FL, Zin WA. Ataques de pânico provocados pelo dióxido de carbono: estudo clínico-fenomenológico/Carbon dioxide-induced panic attacks: clinical-phenomenologic study. Rev Bras Psiquiatr. 2001;23(1):15-20.

6. Ballenger JC, Davidson JRT, Lecrubier Y, Nutt DJ, Baldwin DS, den Bôer JA, et al. Consensus statement on panic disorder from the international consensus group on depression and anxiety. J Clin Psychiatry. 1998;59(8 Suppl):47-54.

7. Oliveira SS, Santos IS, Silva JFP, Machado EC. Prevalência e fatores associados à doença do refluxo gastroesofágico/Gastroesophageal reflux disease: prevalence and associated factors. Arq Gastroenterol. 2005;42(2):116-121.

8. Del-Ben CM, Vilela JAA, Crippa JAS, Hallak JEC, Labate CM, Zuardi AW. Confiabilidade da "entrevista clínica estruturada para o DSM-IV - versão clínica" traduzida para o português. Rev Bras Psiquiatr. 2001;23(3):156-9.

9. Gray J. The neuropsychology of anxiety. Oxford, UK: Oxford University Press; 2000.

10. Graeff, FG. Ansiedade, pânico e o eixo hipotálamo-pituitária-adrenal/Anxiety, panic and the hypothalamic-pituitary-adrenal axis. Rev Bras Psiquiatr. 2007;29 Suppl 1:S3-6.

11. Messas GP, Vallada Filho HP. Aspectos genéticos do transtorno de pânico/The genetics of panic disorder. Rev Psiquiatr Clin. 2001;28(1):12-5.

12. Ramos, RT. As bases biológicas do transtorno de pânico/The biological basis of panic disorder. Rev Psiquiatr Clin. 2001;28(1):9-11.

13. Pinheiro SH, Zangrossi-Jr H, Del-Ben CM, Graeff FG. Elevated mazes as animal models of anxiety: effects of serotonergic agents. An Acad Bras Cienc. 2007;79(1):71-85.

14. Menezes GB, Fontenelle LF, Mululo S, Versiani M. Resistência ao tratamento nos transtornos de ansiedade: fobia social, transtorno de ansiedade generalizada e transtorno do pânico/Treatment-resistant anxiety disorders: social phobia, generalized anxiety disorder and panic disorder. Rev Bras Psiquiatr. 2007:29 Suppl 2:S55-60. 